\title{
Regularity and Exponential Growth of Pullback Attractors for Semilinear Parabolic Equations Involving the Grushin Operator
}

\author{
Nguyen Dinh Binh \\ Faculty of Applied Mathematics and Informatics, Hanoi University of Technology, 1 Dai Co Viet, \\ Hai Ba Trung, Hanoi, Vietnam \\ Correspondence should be addressed to Nguyen Dinh Binh, binhngd-fami@mail.hut.edu.vn \\ Received 24 November 2011; Accepted 28 December 2011 \\ Academic Editor: Shaoyong Lai \\ Copyright (c) 2012 Nguyen Dinh Binh. This is an open access article distributed under the Creative \\ Commons Attribution License, which permits unrestricted use, distribution, and reproduction in \\ any medium, provided the original work is properly cited. \\ Considered here is the first initial boundary value problem for a semilinear degenerate parabolic \\ equation involving the Grushin operator in a bounded domain $\Omega$. We prove the regularity and \\ exponential growth of a pullback attractor in the space $S_{0}^{2}(\Omega) \cap L^{2 p-2}(\Omega)$ for the nonautonomous \\ dynamical system associated to the problem. The obtained results seem to be optimal and, in \\ particular, improve and extend some recent results on pullback attractors for reaction-diffusion \\ equations in bounded domains.
}

\section{Introduction}

Let $\Omega$ be a bounded domain in $\mathbb{R}^{N_{1}} \times \mathbb{R}^{N_{2}}\left(N_{1}, N_{2} \geq 1\right)$, with smooth boundary $\partial \Omega$. In this paper, we consider the following problem:

$$
\begin{gathered}
u_{t}-G_{s} u+f(u)=g(t, x), \quad(t, x) \in Q_{\tau, T}=(\tau, T] \times \Omega, \\
u(x, t)=0, \quad x \in \partial \Omega, t \in(\tau, T], \\
u(x, \tau)=u_{\tau}(x), \quad x \in \Omega,
\end{gathered}
$$

where

$$
G_{s} u=\Delta_{x_{1}} u+\left|x_{1}\right|^{2 s} \Delta_{x_{2}} u, \quad x=\left(x_{1}, x_{2}\right) \in \Omega \subset \mathbb{R}^{N_{1}} \times \mathbb{R}^{N_{2}}, s \geqslant 0,
$$


is the Grushin operator, $u_{\tau} \in L^{2}(\Omega)$ is given, the nonlinearity $f$ and the external force $g$ satisfy the following conditions.

(H1) The nonlinearity $f \in C^{1}(\mathbb{R}, \mathbb{R})$ satisfies

$$
\begin{gathered}
f(u) u \geq C_{1}|u|^{p}-C_{2}, \quad p \geq 2, \\
\left|f^{\prime}(u)\right| \leq C_{3}|u|^{p-2}+C_{4}, \\
f^{\prime}(u) \geq-\ell,
\end{gathered}
$$

where $\ell, C_{i},(i=1,2,3,4)$ are positive constants. Relation (1.3) and (1.4) imply that

$$
\alpha_{1}|u|^{p}-\alpha_{2} \leq F(u) \leq \alpha_{3}|u|^{p}+\alpha_{4}
$$

where $F(s)=\int_{0}^{s} f(\tau) d \tau$, and $\alpha_{i}(i=1,2,3,4)$ are positive constants.

(H2) $g \in W_{\text {loc }}^{1,2}\left(\mathbb{R} ; L^{2}(\Omega)\right)$ satisfies

$$
\int_{-\infty}^{t} e^{\lambda_{1} s}\left(\|g(s)\|_{L^{2}(\Omega)}^{2}+\left\|g^{\prime}(s)\right\|_{L^{2}(\Omega)}^{2}\right) d s<+\infty, \quad \forall t \in \mathbb{R}
$$

where $\lambda_{1}$ is the first eigenvalue of the operator $-G_{s}$ in $\Omega$ with the homogeneous Dirichlet boundary condition.

The Grushin operator $G_{s}$ was first introduced in [1]. Noting that if $s>0$, then $G_{s}$ is not elliptic in domains of $\mathbb{R}^{N_{1}} \times \mathbb{R}^{N_{2}}$ which intersect the hyperplane $\left\{x_{1}=0\right\}$. In the last few years, the existence and long-time behavior of solutions to parabolic equations involving the Grushin operator have been studied widely in both autonomous and nonautonomous cases (see, e.g., [2-7]). In particular, the existence of a pullback attractor in $S_{0}^{1}(\Omega) \cap L^{p}(\Omega)$ for the process associated to problem (1.1) is considered in [2].

In this paper we continue the study in the paper [2]. First, we will prove the existence of pullback attractors in $S_{0}^{2}(\Omega)$ (see Section 2 for its definition) and $L^{2 p-2}(\Omega)$. As we know, if the external force $g$ is only in $L^{2}(\Omega)$, then solutions of problem (1.1) are at most in $L^{2 p-2}(\Omega) \cap S_{0}^{2}(\Omega)$ and have no higher regularity. Therefore, there are no compact embedding results that hold for this case. To overcome the difficulty caused by the lack of embedding results, we exploit the asymptotic a priori estimate method which was initiated in $[8,9]$ for autonomous equations and developed recently for nonautonomous equations in the case of pullback attractors in [10]. Noting that, to prove the existence of pullback attractors in $S_{0}^{1}(\Omega) \cap L^{p}(\Omega)$, we only need assumption (H2) of the external force $g$; however, to prove the existence of pullback attractors in $S_{0}^{2}(\Omega)$ and $L^{2 p-2}(\Omega)$, we need an additional assumption of $g$, namely, (3.18) in Section 3. Next, following the general lines of the approach in [11], we give exponential growth conditions in $S_{0}^{2}(\Omega) \cap L^{2 p-2}(\Omega)$ for the pullback attractors. It is noticed that, as far as we know, the best known results on the pullback attractors for nonautonomous reaction-diffusion equations are the boundedness and exponential growth in $H^{2}(\Omega)$ of the pullback attractors $[11,12]$. Therefore, the obtained results seem to be optimal and, in particular when $s=0$, improve the recent results on pullback attractors for the nonautonomous reaction-diffusion equations in [11-15]. 
The content of the paper is as follows. In Section 2, for the convenience of the reader, we recall some concepts and results on function spaces and pullback attractors which we will use. In Section 3, we prove the existence of pullback attractors in the spaces $S_{0}^{2}(\Omega)$ and $L^{2 p-2}(\Omega)$ by using the asymptotic a priori estimate method. In Section 4 , under additional assumptions of $g$, an exponential growth in $S_{0}^{2}(\Omega) \cap L^{2 p-2}(\Omega)$ for the pullback attractors is deduced.

\section{Preliminaries}

\subsection{Operator and Function Spaces}

In order to study the boundary value problem for equations involving the Grushin operator, we have usually used the natural energy space $S_{0}^{1}(\Omega)$ defined as the completion of $C_{0}^{\infty}(\Omega)$ in the following norm:

$$
\|u\|_{S_{0}^{1}(\Omega)}=\left(\int_{\Omega}\left(\left|\nabla_{x_{1}} u\right|^{2}+\left|x_{1}\right|^{2 s}\left|\nabla_{x_{2}} u\right|^{2}\right) d x\right)^{1 / 2}
$$

and the scalar product

$$
((u, v)):=\left(\int_{\Omega}\left(\nabla_{x_{1}} u \nabla_{x_{1}} v+\left|x_{1}\right|^{2 s} \nabla_{x_{2}} u \nabla_{x_{2}} v\right) d x\right)^{1 / 2} .
$$

The following lemma comes from [16].

Lemma 2.1. Assume that $\Omega$ is a bounded domain in $\mathbb{R}^{N_{1}} \times \mathbb{R}^{N_{2}}\left(N_{1}, N_{2} \geq 0\right)$. Then the following embeddings hold:

(i) $S_{0}^{1}(\Omega) \hookrightarrow L^{2_{\alpha}^{*}}(\Omega)$ continuously;

(ii) $S_{0}^{1}(\Omega) \hookrightarrow L^{p}(\Omega)$ compactly if $p \in\left[1,2_{s}^{*}\right)$,

where $2_{s}^{*}=2 N(s) /(N(s)-2), N(s)=N_{1}+(s+1) N_{2}$.

Now, we introduce the space $S_{0}^{2}(\Omega)$ defined as the closure of $C_{0}^{\infty}(\Omega)$ with the norm

$$
\|u\|_{S_{0}^{2}(\Omega)}=\left(\int_{\Omega}\left(\left|\Delta_{x_{1}} u\right|^{2}+\left|x_{1}\right|^{2 \mathrm{~s}}\left|\Delta_{x_{2}} u\right|^{2}\right) d x\right)^{1 / 2}=\left(\int_{\Omega}\left|\mathrm{G}_{\mathrm{s}} u\right|^{2} d x\right)^{1 / 2} .
$$

The following lemma comes directly from the definitions of $S_{0}^{1}(\Omega)$ and $S_{0}^{2}(\Omega)$.

Lemma 2.2. Assume that $\Omega$ is a bounded domain in $\mathbb{R}^{N_{1}} \times \mathbb{R}^{N_{2}}\left(N_{1}, N_{2} \geq 0\right)$, with smooth boundary $\partial \Omega$. Then $S_{0}^{2}(\Omega) \subset S_{0}^{1}(\Omega)$ continuously.

It is known that (see, e.g., [3]) for the operator $A=-G_{s}$, there exist $\left\{e_{j}\right\}_{j \geq 1}$ 
such that

$$
\begin{gathered}
\left(e_{j}, e_{k}\right)=\delta_{j k}, \quad A e_{j}=\lambda_{j} e_{j}, \quad j, k=1,2, \ldots, \\
0<\lambda_{1} \leq \lambda_{2} \leq \lambda_{3} \leq \cdots, \quad \lambda_{j} \longrightarrow+\infty \quad \text { as } j \longrightarrow \infty,
\end{gathered}
$$

and $\left\{e_{j}\right\}_{j \geq 1}$ is a complete orthonormal system in $L^{2}(\Omega)$.

\subsection{Pullback Attractors}

Let $X$ be a Banach space with the norm $\|\cdot\| \cdot \mathcal{B}(X)$ denotes all bounded sets of $X$. The Hausdorff semidistance between $A$ and $B$ is defined by

$$
\operatorname{dist}(A, B)=\sup _{x \in A} \inf _{y \in B}\|x-y\|
$$

Let $\{U(t, \tau): t \geq \tau, \tau \in \mathbb{R}\}$ be a process in $X$, that is, $U(t, \tau): X \rightarrow X$ such that $U(\tau, \tau)=I d$ and $U(t, s) U(s, \tau)=U(t, \tau)$ for all $t \geq s \geq \tau, \tau \in \mathbb{R}$. The process $\{U(t, \tau)\}$ is said to be normto-weak continuous if $U(t, \tau) x_{n} \rightarrow U(t, \tau) x$, as $x_{n} \rightarrow x$ in $X$, for all $t \geq \tau, \tau \in \mathbb{R}$. The following result is useful for proving the norm-to-weak continuity of a process.

Proposition 2.3 (see [9]). Let $X, Y$ be two Banach spaces, and let $X^{*}, Y^{*}$ be, respectively, their dual spaces. Suppose that $X$ is dense in $Y$, the injection $i: X \rightarrow Y$ is continuous, and its adjoint $i^{*}: Y^{*} \rightarrow$ $X^{*}$ is dense, and $\{U(t, \tau)\}$ is a continuous or weak continuous process on $Y$. Then $\{U(t, \tau)\}$ is normto-weak continuous on $X$ if and only if for $t \geq \tau, \tau \in \mathbb{R}, U(t, \tau)$ maps compact sets of $X$ into bounded sets of $X$.

Definition 2.4. The process $\{U(t, \tau)\}$ is said to be pullback asymptotically compact if for any $t \in \mathbb{R}$, any $D \in \mathbb{B}(X)$, any sequence $\tau_{n} \rightarrow-\infty$, and any sequence $\left\{x_{n}\right\} \subset D$, the sequence $\left\{U\left(t, \tau_{n}\right) x_{n}\right\}$ is relatively compact in $X$.

Definition 2.5. A family of bounded sets $B=\{B(t): t \in \mathbb{R}\} \subset X$ is called a pullback absorbing set for the process $\{U(t, \tau)\}$ if for any $t \in \mathbb{R}$ and any $D \in B(X)$, there exist $\tau_{0}=\tau_{0}(D, t) \leq t$ and $B(t) \in \mathbb{B}$ such that

$$
\bigcup_{\tau \leq \tau_{0}} U(t, \tau) D \subset B(t)
$$

Definition 2.6. The family $\mathscr{A}=\{A(t): t \in \mathbb{R}\} \subset \mathbb{B}(X)$ is said to be a pullback attractor for $\{U(t, \tau)\}$ if

(1) $A(t)$ is compact for all $t \in \mathbb{R}$,

(2) A is invariant, that is,

$$
U(t, \tau) A(\tau)=A(t), \quad \forall t \geq \tau,
$$


(3) A is pullback attracting, that is,

$$
\lim _{\tau \rightarrow-\infty} \operatorname{dist}(U(t, \tau) D, A(t))=0, \quad \forall D \in B(X), \text { and all } t \in \mathbb{R},
$$

(4) if $\{C(t): t \in \mathbb{R}\}$ is another family of closed pullback attracting sets, then $A(t) \subset C(t)$, for all $t \in \mathbb{R}$.

Theorem 2.7 (see [13]). Let $\{U(t, \tau)\}$ be a norm-to-weak continuous process which is pullback asymptotically compact. If there exists a pullback absorbing set $B=\{B(t): t \in \mathbb{R}\}$, then $\{U(t, \tau)\}$ has a unique pullback attractor $\mathcal{A}=\{A(t): t \in \mathbb{R}\}$ and

$$
A(t)=\bigcap_{s \leq t} \overline{\bigcup_{\tau \leq s} U(t, \tau) B(\tau)}
$$

In the rest of the paper, we denote by $|\cdot|_{2},(\cdot, \cdot)$ the norm and inner product in $L^{2}(\Omega)$, respectively, and by $|\cdot|_{p}$ the norm in $L^{p}(\Omega)$. By $\|\cdot\|$ we denote the norm in $S_{0}^{1}(\Omega)$. For a Banach space $E,\|\cdot\|_{E}$ will be the norm. We also denote by $C$ an arbitrary constant, which is different from line to line, and even in the same line.

\section{Existence of Pullback Attractors in $S_{0}^{2}(\Omega) \cap L^{2 p-2}(\Omega)$}

It is well known (see, e.g., [2] or [14]) that under conditions $(H 1)-(H 2)$, problem (1.1) defines a process

$$
U(t, \tau): L^{2}(\Omega) \longrightarrow S_{0}^{1}(\Omega) \cap L^{p}(\Omega), \quad \forall t \geq \tau,
$$

where $U(t, \tau) u_{\tau}$ is the unique weak solution of (1.1) with initial datum $u_{\tau}$ at time $\tau$. The process $\{U(t, \tau)\}$ has a pullback attractor in $S_{0}^{1}(\Omega) \cap L^{p}(\Omega)$.

In this section, we will prove that the pullback attractor is in fact in $S_{0}^{2}(\Omega) \cap L^{2 p-2}(\Omega)$.

Lemma 3.1. Assuming that $f$ and $g$ satisfy (H 1)-(H 2), $u(t)$ is a weak solution of (1.1). Then the following inequality holds for $t>\tau$ :

$$
\|u\|^{2}+|u|_{p}^{p} \leq C\left(e^{-\lambda_{1}(t-\tau)}\left|u_{\tau}\right|_{2}^{2}+1+e^{-\lambda_{1} t} \int_{-\infty}^{t} e^{\lambda_{1} s}|g(s)|_{2}^{2} d s\right)
$$

where $C$ is a positive constant.

Proof. Multiplying (1.1) by $u$ and then integrating over $\Omega$, we get

$$
\frac{1}{2} \frac{d}{d t}|u|_{2}^{2}+\|u\|^{2}+\int_{\Omega} f(u) u d x=\int_{\Omega} g(t) u d x \leq \frac{1}{\lambda_{1}}|g(t)|_{2}^{2}+\frac{\lambda_{1}}{4}|u|_{2}^{2} .
$$


Using hypothesis (H1) and the inequality $\|u\|^{2} \geq \lambda_{1}|u|_{2}^{2}$, we have

$$
\frac{d}{d t}|u|_{2}^{2}+\lambda_{1}|u|_{2}^{2}+C\left(\|u\|^{2}+|u|_{p}^{p}\right) \leq C\left(1+|g(t)|_{2}^{2}\right)
$$

Letting $F(s)=\int_{0}^{s} f(\tau) d \tau$, by $(H 1)$, we have

$$
\alpha_{1}|u|^{p}-\alpha_{2} \leq F(u) \leq \alpha_{3}|u|^{p}+\alpha_{4}
$$

Now multiplying (3.4) by $e^{\lambda_{1} t}$ and using (3.5), we get

$$
\frac{d}{d t}\left(e^{\lambda_{1} t}|u(t)|_{2}^{2}\right)+C e^{\lambda_{1} t}\left(\|u\|^{2}+2 \int_{\Omega} F(u(t)) d x\right) \leq C e^{\lambda_{1} t}\left(1+|g(t)|_{2}^{2}\right)
$$

Integrating (3.6) from $\tau$ to $s \in[\tau, t-1]$ and $s$ to $s+1$, respectively, we obtain

$$
\begin{gathered}
e^{\lambda_{1} s}|u(s)|_{2}^{2} \leq e^{\lambda_{1} \tau}\left|u_{\tau}\right|_{2}^{2}+C e^{\lambda_{1} s}+C \int_{\tau}^{s} e^{\lambda_{1} r}|g(r)|_{2}^{2} d r, \quad \forall s \in[\tau, t-1], \\
C \int_{s}^{s+1} e^{\lambda_{1} r}\left(\|u(r)\|^{2}+2 \int_{\Omega} F(u(x, r)) d x\right) d r \\
\leq e^{\lambda_{1} s}|u(s)|_{2}^{2}+C \int_{s}^{s+1} e^{\lambda_{1} r}\left(1+|g(r)|_{2}^{2}\right) d r \\
\leq e^{\lambda_{1} \tau}\left|u_{\tau}\right|_{2}^{2}+C e^{\lambda_{1} s}+C \int_{\tau}^{s} e^{\lambda_{1} r}|g(r)|_{2}^{2} d r \\
+C e^{\lambda_{1}(s+1)}+C \int_{s}^{s+1} e^{\lambda_{1} r}|g(r)|_{2}^{2} d r \\
\leq C\left(e^{\lambda_{1} \tau}\left|u_{\tau}\right|_{2}^{2}+e^{\lambda_{1} t}+\int_{\tau}^{t} e^{\lambda_{1} r}|g(r)|_{2}^{2} d r\right) .
\end{gathered}
$$

Multiplying (1.1) by $u_{t}$ and integrating over $\Omega$, we have

$$
\left|u_{t}(s)\right|_{2}^{2}+\frac{1}{2} \frac{d}{d s}\left(\|u(s)\|^{2}+2 \int_{\Omega} F(u(x, s)) d x\right)=\int_{\Omega} g(s) u_{t}(s) \leq \frac{1}{2}|g(s)|_{2}^{2}+\frac{1}{2}\left|u_{t}(s)\right|_{2}^{2}
$$

Thus

$$
\begin{aligned}
e^{\lambda_{1} s}\left|u_{t}(s)\right|_{2}^{2}+\frac{d}{d s}\left[e^{\lambda_{1} s}\left(\|u(s)\|^{2}+2 \int_{\Omega} F(u(x, s)) d x\right)\right] \\
\leq \lambda_{1} e^{\lambda_{1} s}\left(\|u(s)\|^{2}+2 \int_{\Omega} F(u(x, s)) d x\right)+e^{\lambda_{1} s}|g(s)|_{2}^{2} .
\end{aligned}
$$


Combining (3.8) and (3.10), and using the uniform Gronwall inequality, we have

$$
e^{\lambda_{1} t}\left(\|u(t)\|^{2}+2 \int_{\Omega} F(u(x, t)) d x\right) \leq C\left(e^{\lambda_{1} \tau}\left|u_{\tau}\right|^{2}+e^{\lambda_{1} t}+\int_{-\infty}^{t} e^{\lambda_{1} s}|g(s)|_{2}^{2} d s\right) .
$$

Using (H1) once again and thanks to $e^{\lambda_{1} \tau}\left|u_{\tau}\right|_{2}^{2} \rightarrow 0$ as $\tau \rightarrow-\infty$, we get the desired result from (3.11).

Lemma 3.2. Assume that (H 1), (H 2) hold. Then for any $t \in \mathbb{R}$ and any $D \subset L^{2}(\Omega)$ that is bounded, there exists $\tau_{0} \leq t-1$ such that

$$
\left|u_{t}(t)\right|_{2}^{2} \leq C\left(1+e^{-\lambda_{1} t} \int_{-\infty}^{t} e^{\lambda_{1} s}\left(|g(s)|_{2}^{2}+\left|g^{\prime}(s)\right|_{2}^{2}\right) d s\right)
$$

for any $\tau \leq \tau_{0}$ and any $u_{\tau} \in D$, where $u_{t}(s)=\left.(d / d t)\left(U(t, \tau) u_{\tau}\right)\right|_{t=s}$.

Proof. Integrating (3.10) from $r$ to $r+1, r \in[\tau, t-1]$ and using (3.8) and (3.11), in particular we find

$$
\begin{aligned}
\int_{r}^{r+1} e^{\lambda_{1} s}\left|u_{t}\right|_{2}^{2} d s \leq & e^{\lambda_{1} r}\left(\|u(r)\|^{2}+2 \int_{\Omega} F(u(x, r)) d x\right) \\
& +\lambda_{1} \int_{r}^{r+1} e^{\lambda_{1} s}\left(\|u(s)\|^{2}+2 \int_{\Omega} F(u(x, s)) d x\right) d s \\
& +\int_{r}^{r+1} e^{\lambda_{1} s}|g(s)|_{2}^{2} d s \leq C\left(e^{\lambda_{1} \tau}\left|u_{\tau}\right|_{2}^{2}+e^{\lambda_{1} t}+\int_{-\infty}^{t} e^{\lambda_{1} s}|g(s)|_{2}^{2} d s\right) .
\end{aligned}
$$

On the other hand, differentiating (1.1) and denoting $v=u_{t}$, we have

$$
v_{t}-G_{s} v+f^{\prime}(u) v=g^{\prime}(t) .
$$

Taking the inner product of (3.14) with $v$ in $L^{2}(\Omega)$, we get

$$
\frac{1}{2} \frac{d}{d t}|v|_{2}^{2}+\|v\|^{2}+\left(f^{\prime}(u) v, v\right)=\left(g^{\prime}(t), v\right)
$$

Using (1.5) and Young's inequality, after a few computations, we see that

$$
\frac{d}{d t}\left(e^{\lambda_{1} t}|v|_{2}^{2}\right)+2 e^{\lambda_{1} t}\|v\|^{2} \leq C e^{\lambda_{1} t}|v|_{2}^{2}+C e^{\lambda_{1} t}\left|g^{\prime}(t)\right|_{2}^{2}
$$

Combining (3.16) and (3.13) and using the uniform Gronwall inequality, we obtain

$$
e^{\lambda_{1} t}|v(t)|_{2}^{2} \leq C\left(e^{\lambda_{1} \tau}\left|u_{\tau}\right|_{2}^{2}+e^{\lambda_{1} t}+\int_{-\infty}^{t} e^{\lambda_{1} s}\left(|g(s)|_{2}^{2}+\left|g^{\prime}(s)\right|_{2}^{2}\right) d s\right) .
$$

The proof is now complete because $e^{\lambda_{1} \tau}\left|u_{\tau}\right|_{2}^{2} \rightarrow 0$ as $\tau \rightarrow-\infty$. 


\subsection{Existence of a Pullback Attractor in $L^{2 p-2}(\Omega)$}

In this section, following the general lines of the method introduced in [9], we prove the existence of a pullback attractor in $L^{2 p-2}(\Omega)$. In order to do this, we need an additional condition of $g$

$$
\int_{-\infty}^{t} e^{\lambda_{1} t}\left\|g^{\prime}(t)\right\|_{L^{m}(\Omega)}^{m^{\prime}} d t<+\infty, \quad \forall \mathrm{t} \in \mathbb{R}
$$

where $m, m^{\prime}$ are defined as in (3.30).

Lemma 3.3. The process $\{U(t, \tau)\}$ associated to problem (1.1) has a pullback absorbing set in $L^{2 p-2}(\Omega)$.

Proof. Multiplying (1.1) by $|u|^{p-2} u$ and integrating over $\Omega$, we get

$$
\begin{aligned}
& \int_{\Omega} u_{t}|u|^{p-2} u d x+(p-1) \int_{\Omega}\left(\left|\nabla_{x_{1}} u\right|^{2}+\left|x_{1}\right|^{2 s}\left|\nabla_{x_{2}} u\right|^{2}\right)|u|^{p-2} d x+\left.\int_{\Omega} f(u)|| u\right|^{p-2} u d x \mid \\
& =\int_{\Omega}|g(t) u|^{p-2} u d x .
\end{aligned}
$$

From (1.3) and the fact that $L^{p}(\Omega) \subset L^{p-2}(\Omega)$ continuously, we have

$$
\int_{\Omega} f(u)|u|^{p-2} u d x \geq \int_{\Omega}\left(C_{1}|u|^{p}-C_{2}\right)|u|^{p-2} d x \geq C_{1}\|u\|_{L^{2 p-2}(\Omega)}^{2 p-2}-C_{2}|u|_{p}^{p} .
$$

On the other hand, by Cauchy's inequality, we see that

$$
\begin{gathered}
\left.\left.\left|\int_{\Omega} u_{t}\right| u\right|^{p-2} u d x\left|\leq \frac{C_{1}}{4}\|u\|_{L^{2 p-2}(\Omega)}^{2 p-2}+\frac{1}{C_{1}}\right| u_{t}\right|_{2} ^{2} \\
\left.\left.\left|\int_{\Omega} g(t)\right| u\right|^{p-2} u d x\left|\leq \frac{C_{1}}{4}\|u\|_{L^{2 p-2}(\Omega)}^{2 p-2}+\frac{1}{4}\right| g(t)\right|_{2} ^{2} .
\end{gathered}
$$

Combining (3.19)-(3.22) imply that

$$
\|u\|_{L^{2 p-2}(\Omega)}^{2 p-2} \leq C\left(\left|u_{t}\right|_{2}^{2}+|u|_{p}^{p}+|g(t)|_{2}^{2}\right)
$$

Applying (3.2) and Lemma 3.2, we conclude the existence of a pullback absorbing set in $L^{2 p-2}(\Omega)$ for the process $U(t, \tau)$. 
Lemma 3.4. For any $s \in \mathbb{R}$, any $2 \leq p<\infty$, and any bounded set $B \subset L^{2}(\Omega)$, there exists $\tau_{0}$ such that

$$
\int_{\Omega}\left|u_{t}(s)\right|^{p} d x \leq M, \quad \forall \tau \leq \tau_{0}, u_{\tau} \in B
$$

where $M$ depends on $s, p$ but not on $B$, and $u_{t}(s)=\left.(d / d t)\left(U(t, \tau) u_{\tau}\right)\right|_{t=s}$.

Proof. We will prove the lemma by induction argument. Letting $\beta=N(s) /(N(s)-2)>1$ and denoting $v=u_{t}$ we prove that for $k=0,1,2, \ldots$, there exist $\tau_{k}$ and $M_{k}(s)$ such that

$$
\begin{gathered}
e^{\lambda_{1} s} \int_{\Omega}|v(s)|^{2 \beta^{k}} d x \leq M_{k}(\mathrm{~s}) \quad \text { for any } u_{\tau} \in B, \tau \leq \tau_{k}, \\
\int_{s}^{s+1}\left(e^{\lambda_{1} r} \int_{\Omega}|v(r)|^{2 \beta^{k+1}} d x\right)^{1 / \beta} d r \leq M_{k}(s) \quad \text { for any } u_{\tau} \in B, \tau \leq \tau_{k},
\end{gathered}
$$

where $\tau_{k}$ depends on $k$ and $B$ and $M_{k}$ depends only on $k$.

For $k=0$, we have $\left(P_{0}\right)$ from (3.17). Integrating (3.16) and using $S_{0}^{1}(\Omega) \hookrightarrow L^{2 \beta}(\Omega)$ continuously, we get $\left(Q_{0}\right)$.

Assuming that $\left(P_{k}\right),\left(Q_{k}\right)$ hold, we prove so are $\left(P_{k+1}\right)$ and $\left(Q_{k+1}\right)$. Multiplying (3.14) by $|v|^{2 \beta^{k+1}-2} v$ and integrating over $\Omega$, we obtain

$$
\begin{aligned}
& C \frac{d}{d t} \int_{\Omega}|v|^{2 \beta^{k+1}} d x+C \int_{\Omega}\left(\left|\nabla_{x_{1}} v\right|^{2}+\left|x_{1}\right|^{2 s}\left|\nabla_{x_{2}} v\right|^{2}\right)|v|^{\beta^{2 k+1}-2} d x \\
& \quad \leq \ell \int_{\Omega}|v|^{2 \beta^{k+1}} d x+\left(g^{\prime}(t),|v|^{2 \beta^{k+1}-2} v\right) .
\end{aligned}
$$

Using the imbedding $S_{0}^{1}(\Omega) \hookrightarrow L^{2 \beta}(\Omega)$ once again, we get

$$
\begin{gathered}
\int_{\Omega}\left(\left|\nabla_{x_{1}} v\right|^{2}+\left|x_{1}\right|^{2 s}\left|\nabla_{x_{2}} v\right|^{2}\right)|v|^{\beta^{2 k+1}} d x \geq \int_{\Omega}|v|^{2}|v|^{2 \beta^{2 k+1}-2} d x \\
=\left\|v^{\beta^{k+1}}\right\|_{L^{2 \beta}(\Omega)}^{2}=\left(\int_{\Omega}|v|^{2 \beta^{k+2}} d x\right)^{1 / \beta} .
\end{gathered}
$$

Combining Holder's and Young's inequalities, we see that

$$
\begin{aligned}
\int_{\Omega} g^{\prime}(t)|v|^{2 \beta^{k+1}-2} v d x & \leq\left(\int_{\Omega}\left|g^{\prime}(t)\right|^{m} d x\right)^{1 / m}\left(\int_{\Omega}|v|^{\left(2 \beta^{k+1}-1\right) n} d x\right)^{1 / n} \\
\leq & \frac{\left(\int_{\Omega}\left|g^{\prime}(t)\right|^{m} d x\right)^{m^{\prime} / m}}{m^{\prime}}+\frac{\left(\int_{\Omega}|v|^{\left(2 \beta^{k+1}-1\right) n} d x\right)^{n^{\prime} / n}}{n^{\prime}},
\end{aligned}
$$


where $1 / m+1 / n=1 / m^{\prime}+1 / n^{\prime}=1$. Choose $n, n^{\prime}$ such that

$$
\left(2 \beta^{k+1}-1\right) n=2 \beta^{k+2}, \quad \frac{n^{\prime}}{n}=\frac{1}{\beta^{\prime}}
$$

thus

$$
n=\frac{2 \beta^{k+2}}{2 \beta^{k+1}-1}, \quad n^{\prime}=\frac{2 \beta^{k+1}}{2 \beta^{k+1}-1}
$$

Hence

$$
m=\frac{n}{n-1}=\frac{2 \beta^{k+1}-1}{2 \beta^{k+2}-2 \beta^{k+1}+1}, \quad m^{\prime}=2 \beta^{k+1} .
$$

Then from (3.27), we infer that

$$
\int_{\Omega} g^{\prime}(t)|v|^{2 \beta^{k+1}-2} v d x \leq \frac{1}{m^{\prime}}\left\|g^{\prime}(t)\right\|_{L^{m}(\Omega)}^{m^{\prime}}+\frac{1}{n^{\prime}}\left(\int_{\Omega}|v|^{2 \beta^{k+2}} d x\right)^{1 / \beta}
$$

Applying (3.26) and (3.31) in (3.25), we find that

$$
\frac{d}{d t}\left(e^{\lambda_{1} t} \int_{\Omega}|v|^{2 \beta^{k+1}} d x\right)+C e^{\lambda_{1} t}\left(\int_{\Omega}|v|^{2 \beta^{k+2}} d x\right)^{1 / \beta} \leq C e^{\lambda_{1} t} \int_{\Omega}|v|^{2 \beta^{k+1}} d x+C e^{\lambda_{1} t}\left\|g^{\prime}(t)\right\|_{L^{m}(\Omega)}^{m^{\prime}} .
$$

Combining $\left(Q_{k}\right)$ and (3.32), using the uniform Gronwall inequality and taking into account assumption (3.18), we get $\left(P_{k+1}\right)$. On the other hand, integrating (3.32) from $t$ to $t+1$, we find $\left(Q_{k+1}\right)$. Now since $\beta>1$, and taking $k \geq \log _{\beta} p / 2$, we get the desired estimate.

We will use the following lemma.

Lemma 3.5 (see [15]). If there exists $\sigma>0$ such that $\int_{-\infty}^{t} e^{\sigma s}|\varphi(s)|^{2} d s<\infty$, for all $t \in \mathbb{R}$, then

$$
\lim _{\gamma \rightarrow+\infty} \int_{-\infty}^{t} e^{-\gamma(t-s)}|\varphi(s)|^{2} d s=0, \quad t \in \mathbb{R}
$$

Let $H_{m}=\operatorname{span}\left\{e_{1}, e_{2}, \ldots, e_{m}\right\}$ in $L^{2}(\Omega)$, and let $P_{m}: L^{2}(\Omega) \rightarrow H_{m}$ be the orthogonal projection, where $\left\{e_{i}\right\}_{i=1}^{\infty}$ are the eigenvectors of operator $A=-G_{s}$. For any $u \in L^{2}(\Omega)$, we write

$$
u=P_{m} u+\left(I-P_{m}\right) u=u_{1}+u_{2} .
$$


Lemma 3.6. For any $t \in \mathbb{R}$, any $B \subset L^{2}(\Omega)$ and any $\varepsilon$, there exist $\tau_{0}(t, B, \varepsilon)$ and $m_{0} \in \mathbb{N}$ such that

$$
\left|\left(I-P_{m}\right) v\right|_{2}^{2}<\varepsilon, \quad \forall \tau \leq \tau_{0}, \forall u_{\tau} \in B, m \geq m_{0} .
$$

Proof. Multiplying (3.14) by $v_{2}=\left(I-P_{m}\right) v$ and then integrating over $\Omega$, using $\left|\nabla v_{2}\right|_{2}^{2} \geq \lambda_{m}\left|v_{2}\right|_{2}^{2}$ and Cauchy's inequality we get

$$
\frac{d}{d t}\left|v_{2}\right|_{2}^{2}+\lambda_{m}\left|v_{2}\right|_{2}^{2} \leq C \int_{\Omega}\left|f^{\prime}(u) v\right|^{2} d x+C\left|g^{\prime}(t)\right|_{2}^{2}
$$

We multiply (3.36) by $e^{\lambda_{m}} t$ and use assumption (1.4). We get

$$
\frac{d}{d t}\left(e^{\lambda_{m} t}\left|v_{2}\right|_{2}^{2}\right) \leq C e^{\lambda_{m} t} \int_{\Omega}|u|^{2(p-2)}|v|^{2} d x+C e^{\lambda_{m} t}\left|g^{\prime}(t)\right|_{2}^{2}
$$

Integrating (3.37) from $s$ to $t$,

$$
\begin{aligned}
e^{\lambda_{m} t}\left|v_{2}(t)\right|_{2}^{2} & \leq e^{\lambda_{m} s}\left|v_{2}(s)\right|_{2}^{2}+C \int_{s}^{t} e^{\lambda_{m} r} \int_{\Omega}|u|^{2(p-2)}|v|^{2} d x d r+C \int_{s}^{t} e^{\lambda_{m} r}\left|g^{\prime}(r)\right|_{2}^{2} d r \\
& \leq e^{\lambda_{m} s}|v(s)|_{2}^{2}+C \int_{-\infty}^{t} e^{\lambda_{m} r} \int_{\Omega}|u|^{2(p-2)}|v|^{2} d x d r+C \int_{-\infty}^{t} e^{\lambda_{m} r}\left|g^{\prime}(r)\right|_{2}^{2} d r
\end{aligned}
$$

Now integrating (3.38) with respect to $s$ from $\tau$ to $t$, we infer that

$$
\begin{gathered}
(t-\tau) e^{\lambda_{m} t}\left|v_{2}(t)\right|_{2}^{2} \leq \int_{\tau}^{t} e^{\lambda_{m} r}|v(r)|_{2}^{2} d r+C(t-\tau) \int_{-\infty}^{t} e^{\lambda_{m} r} \int_{\Omega}|u|^{2(p-2)}|v|^{2} d x d r \\
+C(t-\tau) \int_{-\infty}^{t} e^{\lambda_{m} r}\left|g^{\prime}(r)\right|_{2}^{2} d r .
\end{gathered}
$$

Thus

$$
\begin{aligned}
\left|v_{2}(t)\right|_{2}^{2} \leq & \frac{1}{t-\tau} \int_{-\infty}^{t} e^{-\lambda_{m}(t-r)}|v(r)|_{2}^{2} d r+C \int_{-\infty}^{t} e^{-\lambda_{m}(t-r)} \int_{\Omega}|u|^{2(p-2)}|v|^{2} d x d r \\
& +C \int_{-\infty}^{t} e^{-\lambda_{m}(t-r)}\left|g^{\prime}(r)\right|_{2}^{2} d r .
\end{aligned}
$$

By Lemma 3.5 and since $\lambda_{m} \rightarrow+\infty$ as $m \rightarrow+\infty$, there exist $\tau_{1}$ and $m_{1}$ such that

$$
\begin{gathered}
\frac{1}{t-\tau} \int_{-\infty}^{t} e^{-\lambda_{m}(t-r)} C \int_{-\infty}^{t} e^{-\lambda_{m}(t-r)}\left|g^{\prime}(r)\right|_{2}^{2} d r<\frac{\varepsilon}{3}, \\
|v(r)|_{2}^{2} d r<\frac{\varepsilon}{3},
\end{gathered}
$$


for all $\tau \leq \tau_{1}$ and $m \geq m_{1}$. For the second term of the right-hand side of (3.40), using Holder's inequality we have

$$
\begin{aligned}
& \int_{-\infty}^{t} e^{-\lambda_{m}(t-r)} \int_{\Omega}|u|^{2(p-2)}|v|^{2} d x d r \\
& \quad \leq \int_{-\infty}^{t}\left(\int_{\Omega} e^{\left((-p-1) /(p-2)_{\lambda_{m}}\right)(t-r)}|u|^{2 p-2} d x\right)^{(p-2) /(p-1)}\left(\int_{\Omega} e^{-(p-1) \lambda_{m}(t-r)}|v|^{2 p-2} d x\right)^{1 /(p-1)} d r \\
& \quad \leq\left(\int_{-\infty}^{t} e^{\left((-p-1) /(p-2)_{\lambda_{m}}\right)(t-r)}\|u\|_{L^{2 p-2}(\Omega)}^{2 p-2} d r\right)^{(p-2) /(p-1)}\left(\int_{-\infty}^{t} e^{-(p-1) \lambda_{m}(t-r)} d r \int_{\Omega}|v|^{2 p-2} d x\right)^{1 /(p-1)} .
\end{aligned}
$$

From Lemmas (3.5)-(3.7), we see that there exist $\tau_{2}$ and $m_{2} \in \mathbb{N}$ such that

$$
C \int_{-\infty}^{t} e^{-\lambda_{m}(t-r)} \int_{\Omega}|u|^{2(p-2)}|v|^{2} d x d r<\frac{\varepsilon}{3}, \quad \forall \tau \leq \tau_{0}, m \geq m_{2}
$$

Let $\tau_{0}=\min \left\{\tau_{1}, \tau_{2}\right\}$ and $m_{0}=\max \left\{m_{1}, m_{2}\right\}$, from (3.40), taking into account (3.41) and (3.43), we obtain (3.35).

Lemma 3.7 (see [9]). Let $B$ be a bounded subset in $L^{q}(\Omega)(q \geq 1)$. If $B$ has a finite $\varepsilon$-net in $L^{q}(\Omega)$, then there exists an $M=M(B, \varepsilon)$, such that for any $u \in B$, the following estimate is valid:

$$
\int_{\Omega(|u| \geq M)}|u|^{q} d x<\varepsilon
$$

Using Lemma 3.7 and taking into account Lemmas 3.2 and 3.6 we conclude that the set $\left\{u_{t}(s): s \leq t, u_{\tau} \in B\right\}$ has a finite $\epsilon$-net in $L^{2}(\Omega)$. Therefore, we get the following result.

Lemma 3.8. For any $t \in \mathbb{R}$, any $B \subset L^{2}(\Omega)$ that is bounded, and any $\varepsilon>0$, there exists $\tau_{0} \leq t$ and $M_{0}>0$ such that

$$
\int_{\Omega(|u| \geq M)}\left|u_{t}(t)\right|^{2} d x<\varepsilon, \quad \forall \tau<\tau_{0}, M>M_{0}, u_{\tau} \in B
$$

Lemma 3.9 (see [9]). For any $t \in \mathbb{R}$, any bounded set $B \subset L^{2}(\Omega)$, and any $\varepsilon>0$, there exist $\tau_{0}$ and $M_{0}>0$ such that

$$
\operatorname{mes}(\Omega(u(t) \geq M))<\varepsilon \quad \forall \tau \leq \tau_{0}, M \geq M_{0}, u_{\tau} \in B
$$

where mes is the Lebesgue measure in $\mathbb{R}^{N}$ and $\Omega(u(t) \geq M)=\{x \in \Omega: u(t, x) \geq M\}$.

Lemma 3.10 (see [2]). Let $\{U(t, \tau)\}$ be a norm-to-weak continuous process in $L^{2}(\Omega)$ and $L^{q}(\Omega), q \geq$ 2. Then $\{U(t, \tau)\}$ is pullback asymptotically compact in $L^{q}(\Omega)$ if 
(i) $\{U(t, \tau)\}$ is pullback asymptotically compact in $L^{2}(\Omega)$;

(ii) for any $t \in \mathbb{R}$, any bounded set $D \subset L^{2}(\Omega)$, and any $\epsilon>0$, there exist $M>0$ and $\tau_{0} \leq t$

$$
\sup _{\tau \leq \tau_{0}} \sup _{u_{\tau} \in D}\left(\int_{\Omega\left(\left|U(t, \tau) u_{\tau}\right| \geq M\right)}\left|U(t, \tau) u_{\tau}\right|^{q} d x\right) \leq C \epsilon,
$$

where $C$ is independent of $M, \tau, u_{\tau}$, and $\epsilon$.

We are now ready to prove the existence of a pullback attractor in $L^{2 p-2}(\Omega)$.

Theorem 3.11. Assume that assumptions (1.3)-(1.7) and (3.18) hold. Then the process $\{U(t, \tau)\}$ associated to problem (1.1) possesses a pullback attractor $A_{2 p-2}=\left\{A_{2 p-2}(t)\right\}_{t \in \mathbb{R}}$ in $L^{2 p-2}(\Omega)$.

Proof. Because of Lemma 3.10, since $\{U(t, \tau)\}$ has a pullback absorbing set in $L^{2 p-2}(\Omega)$, we only have to prove that for any $t \in \mathbb{R}$, any $B \subset L^{2}(\Omega)$, and any $\varepsilon>0$, there exist $\tau_{2} \leq t$ and $M_{2}>0$ such that

$$
\int_{\Omega(|u| \geq M)}|u|^{2 p-2} d x \leq C \varepsilon, \quad \forall \tau \leq \tau_{2}, M \geq M_{2}, u_{\tau} \in B .
$$

Taking the inner product of $(1.1)$ with $(u-M)_{+}^{p-1}$ in $L^{2}(\Omega)$, where

$$
(u-M)_{+}= \begin{cases}u-M & \text { if } u \geq M, \\ 0 & \text { if } u<M,\end{cases}
$$

we have

$$
\begin{aligned}
& \int_{\Omega} u_{t}(u-M)_{+}^{p-1} d x+(p-1) \int_{\Omega}\left(\left|\nabla_{x_{1}} u\right|^{2} d x+\left|x_{1}\right|^{2 s}\left|\nabla_{x_{2}} u\right|^{2}\right)(u-M)_{+}^{p-2} d x \\
& \quad+\int_{\Omega} f(u)(u-M)_{+}^{p-1} d x \\
& \quad \leq \int_{\Omega} g(t)(u-M)_{+}^{p-1} d x .
\end{aligned}
$$

Some standard computations give us

$$
\begin{gathered}
\int_{\Omega} f(u)(u-M)_{+}^{p-1} d x \geq C \int_{\Omega(u \geq M)}|u|^{2 p-2} d x+C \int_{\Omega(u \geq M)}|u|^{p} d x, \\
-\int_{\Omega} u_{t}(u-M)_{+}^{p-1} d x \leq \frac{C}{4} \int_{\Omega(u \geq M)}|u|^{2 p-2} d x+\frac{1}{C} \int_{\Omega(u \geq M)}\left|u_{t}\right|^{2} d x, \\
\int_{\Omega} g(t)(u-M)_{+}^{p-1} d x \leq \frac{C}{4} \int_{\Omega(u \geq M)}|u|^{2 p-2} \mathrm{~d} x+\frac{1}{C} \int_{\Omega(u \geq M)}|g(t)|^{2} d x .
\end{gathered}
$$


Combining (3.50)-(3.53), we find

$$
\int_{\Omega(u \geq M)}|u|^{2 p-2} d x \leq C\left(\int_{\Omega(u \geq M)}\left|u_{t}\right|^{2} d x+\int_{\Omega(u \geq M)}|g(t)|^{2} d x+C \int_{\Omega(u \geq M)}|u|^{p} d x\right) .
$$

Applying Lemmas 3.7 and 3.8 to (3.54) we find there exist $\tau_{0}$ and $M_{0}$ such that

$$
\int_{\Omega(u \geq M)}|u|^{2 p-2} d x<\varepsilon \quad \forall \tau \leq \tau_{0}, M \geq M_{0}
$$

Repeating the above arguments with $\left|(u+M)_{-}\right|^{p-2}(u+M)_{-}$in place of $(u-M)_{+}^{p-1}$, we have

$$
\int_{\Omega(u \leq-M)}|u|^{2 p-2} d x<\varepsilon \quad \forall \tau \leq \tau_{1}, M \geq M_{1}
$$

for some $\tau_{1} \leq t$ and $M_{1}>0$, where

$$
(u+M)_{-}= \begin{cases}u+M & \text { if } u \leq-M \\ 0 & \text { if } u>M .\end{cases}
$$

Letting $\tau_{2}=\min \left\{\tau_{0}, \tau_{1}\right\}$ and $M_{2}=\max \left\{M_{0}, M_{1}\right\}$ we have

$$
\int_{\Omega\left(|u| \geq M_{2}\right)}|u|^{2 p-2} d x<C \varepsilon, \quad \forall \tau \leq \tau_{2}, M \geq M_{2} .
$$

This completes the proof.

\subsection{Existence of a Pullback Attractor in $S_{0}^{2}(\Omega)$}

In this section, we prove the existence of a pullback attractor in $S_{0}^{2}(\Omega)$.

Lemma 3.12. The process $\{U(t, \tau)\}$ associated to (1.1) has a pullback absorbing set in $S_{0}^{2}(\Omega)$.

Proof. We multiply (1.1) by $-G_{s} u$; then, using $f(0)=0$, we have

$$
\|u\|_{S_{0}^{2}(\Omega)}^{2}=\int_{\Omega} u_{t} G_{s} u d x-\int_{\Omega} f^{\prime}(u)\left(\left|\nabla_{x_{1}} u\right|^{2}+\left|x_{1}\right|^{2 s}\left|\nabla_{x_{2}} u\right|^{2}\right) d x-\int_{\Omega} g(t) G_{s} u d x
$$

Using $f^{\prime}(u) \geq-\ell$, Cauchy's inequality, and argument as in Lemma 3.3, from (3.59) we have

$$
\|u\|_{S_{0}^{2}(\Omega)}^{2} \leq 2\left(\left|u_{t}\right|_{2}^{2}+\ell\|u\|^{2}+|g(t)|_{2}^{2}\right)
$$

Taking into account (3.11), the proof is complete. 
In order to prove the existence of the pullback attractor in $S_{0}^{2}(\Omega)$, we will verify socalled "(PDC) condition", which is defined as follow

Definition 3.13. A process $\{U(t, \tau)\}$ is said to satisfy (PDC) condition in $X$ if for any $t \in \mathbb{R}$, any bounded set $B \subset L^{2}(\Omega)$ and any $\varepsilon>0$, there exists $\tau_{0} \leq t$ and a finite dimensional subspace $X_{1}$ of $X$ such that

(i) $P\left(\bigcup_{\tau \leq \tau_{0}} U(t, \tau) B\right)$ is bounded in $X$; and

(ii) $\left\|\left(I_{X}-P\right) U(t, \tau) u_{\tau}\right\|_{X}<\varepsilon$, for all $\tau \leq \tau_{0}$ and $u_{\tau} \in B$, where $P: X \rightarrow X_{1}$ is a canonical projection and $I_{X}$ is the identity.

Lemma 3.14 (see [13]). If a process $\{U(t, \tau)\}$ satisfies (PDC) condition in $X$ then it is pullback asymptotically compact in $\mathrm{X}$. Moreover, if $\mathrm{X}$ is convex then the converse is true.

Lemma 3.15 (see [9]). Assume that $f$ satisfies (1.3) and (1.5). Then for any subset $A \subset L^{2 p-2}(\Omega)$, if $\kappa(A)<\varepsilon$ in $L^{2 p-2}(\Omega)$, then we have

$$
\kappa(f(A))<C \varepsilon \text { in } L^{2}(\Omega),
$$

where the Kuratowski noncompactness measure $\mathcal{\kappa}(B)$ in a Banach space X defined as

$$
\kappa(B)=\inf \{\delta: B \text { has a finite covering by balls in } X \text { with radii } \delta\} .
$$

Theorem 3.16. Assume that $f$ satisfies (1.3)-(1.5), $g$ satisfies (1.7) and (3.18). Then the process $\{U(t, \tau)\}$ generated by (1.1) has a pullback attractor $\mathcal{A}=\{A(t): t \in \mathbb{R}\}$ in $S_{0}^{2}(\Omega)$.

Proof. We consider a complete trajectory $u(t)$ lies on pullback attractor $\mathcal{A}_{2 p-2}$ in $L^{2 p-2}(\Omega)$ for $U(t, \tau)$, that is, $u(t) \in \mathcal{A}_{2 p-2}(t)$ and $U(t, \tau) u_{\tau}=u(t)$, for all $t \geq \tau$. Denoting $A=-G_{s}$ and multiplying (1.1) by $A u_{2}=A\left(I-P_{m}\right) u=\left(I-P_{m}\right) A u$ we have

$$
\left(u_{t}, A u_{2}\right)+\left\|u_{2}\right\|_{S_{0}^{2}(\Omega)}^{2}+\int_{\Omega} f(u) A u_{2} d x=\left(g(t), A u_{2}\right) .
$$

Using Holder's inequality we get

$$
\left\|u_{2}\right\|_{S_{0}^{2}(\Omega)}^{2} \leq C\left(\left|\left(I-P_{m}\right) u_{t}\right|_{2}^{2}+\left|\left(I-P_{m}\right) g(t)\right|_{2}^{2}+\int_{\Omega}(f(u))^{2} d x\right) .
$$

Thanks to Lemmas 3.6 and 3.15 and the fact that $g \in C_{\text {loc }}\left(\mathbb{R} ; L^{2}(\Omega)\right)$, we see that $\{U(t, \tau)\}$ satisfies condition (PDC) in $S_{0}^{2}(\Omega)$. Now from Lemmas 3.3 and 3.14 we get the desired result.

\section{Exponential Growth in $S_{0}^{2}(\Omega) \cap L^{2 p-2}(\Omega)$ of Pullback Attractors}

In this section, we will give an exponential growth condition in $S_{0}^{2}(\Omega) \cap L^{2 p-2}(\Omega)$ for the pullback attractor $\mathcal{A}(\tau)$.

First, we recall a result in [17] which is necessary for the proof of our results. 
Lemma 4.1. Let $X, Y$ be Banach spaces such that $X$ is reflexive, and the inclusion $X \subset Y$ is continuous. Assume that $\left\{u_{n}\right\}$ is bounded sequence in $L^{\infty}\left(t_{0}, T ; X\right)$ such that $u_{n} \rightarrow u$ weakly in $L^{q}\left(t_{0}, T ; X\right)$ for some $q \in[1,+\infty)$ and $u \in C^{0}\left(\left[t_{0}, T\right] ; Y\right)$. Then, $u(t) \in X$ for all $t \in\left[t_{0}, T\right]$ and

$$
\|u\|_{X} \leq \sup _{n \geq 1}\left\|u_{n}\right\|_{L^{\infty}\left(t_{0}, T ; X\right)}, \quad \forall t \in\left[t_{0}, T\right] .
$$

In the following theorem, instead of evaluating the functions $u_{n}$ which are differentiable enough and then using Lemma 4.1, we will formally evaluate the function $u$.

Theorem 4.2. Assume that $f$ satisfies (1.3)-(1.5), g satisfies (H 2), (3.18) and the following conditions

$$
\begin{gathered}
\lim _{\tau \rightarrow-\infty} e^{\lambda_{1} \tau} \int_{\tau}^{\tau+1}\left|g^{\prime}(s)\right|_{2}^{2} d s=0, \\
\lim _{\tau \rightarrow-\infty}\left(e^{\lambda_{1} \tau}|g(s)|_{2}^{2}\right)=0 .
\end{gathered}
$$

Then $\mathcal{A}(\tau)$ satisfies

$$
\lim _{\tau \rightarrow-\infty} e^{\lambda_{1} \tau}\left\{\sup _{w \in \mathcal{A}(\tau)}\|w\|_{L^{2 p-2}(\Omega)}^{2 p-2}+\sup _{w \in \mathcal{A}(\tau)}\|w\|_{S_{0}^{2}(\Omega)}^{2}\right\}=0
$$

Proof. We differentiate with respect to time in (1.1), then multiply by $u_{t}$, we get

$$
\begin{aligned}
\frac{1}{2} \frac{d}{d r}\left|u^{\prime}(r)\right|_{2}^{2}+\|u(r)\|^{2} & =-\int_{\Omega} f^{\prime}(u) u^{\prime}(r) u^{\prime}(r) \mathrm{d} x+\int_{\Omega} g^{\prime}(r) u^{\prime}(r) d x \\
& \leq \ell\left|u^{\prime}(r)\right|_{2}^{2}+\frac{1}{2} u^{\prime}(r)_{2}^{2}+\frac{1}{2}\left|g^{\prime}(r)\right|_{2}^{2} .
\end{aligned}
$$

Integrating in the last inequality, in particular, we get

$$
\left|u^{\prime}(r)\right|_{2}^{2} \leq\left|u^{\prime}(s)\right|_{2}^{2}+(2 \ell+1) \int_{\tau+\varepsilon / 2}^{t}\left|u^{\prime}(\theta)\right|_{2}^{2} d \theta+\int_{\tau+\epsilon / 2}^{t}\left|g^{\prime}(\theta)\right|_{2}^{2} d \theta \text {. }
$$

for all $\tau+\epsilon / 2 \leq \mathrm{s} \leq \tau \leq t$. Now, integrating with respect to $s$, between $\tau+\epsilon / 2$ and $r$

$$
\begin{aligned}
\left(r-\tau-\frac{\epsilon}{2}\right)\left|u^{\prime}(r)\right|_{2}^{2} \leq & {\left[\left(r-\tau-\frac{\epsilon}{2}\right)(2 l+1)+1\right] \int_{\tau+\epsilon / 2}^{t}\left|u^{\prime}(\theta)\right|_{2}^{2} d \theta } \\
& +\left(r-\tau-\frac{\epsilon}{2}\right) \int_{\tau+\epsilon / 2}^{t}\left|g^{\prime}(\theta)\right|_{2}^{2} d \theta .
\end{aligned}
$$


for all $\tau+\epsilon / 2 \leq r \leq t$, in paricular,

$$
\begin{aligned}
\left|u^{\prime}(r)\right|_{2}^{2} \leq & 2 \epsilon^{-1}\left[\left(r-\tau-\frac{\epsilon}{2}\right)(2 l+1)+1\right] \int_{\tau+\epsilon / 2}^{t}\left|u^{\prime}(\theta)\right|_{2}^{2} d \theta \\
& +\int_{\tau+\epsilon / 2}^{t}\left|g^{\prime}(\theta)\right|_{2}^{2} d \theta
\end{aligned}
$$

for all $r \in[\tau+\epsilon, t]$.

Multiplying (1.1) by $u$ and then integrating on $\Omega$, we get

$$
\frac{1}{2} \frac{d}{d t}|u|_{2}^{2}+\|u\|^{2}+\int_{\Omega} f(u) u d x=\int_{\Omega} g(t) u d x \leq \frac{1}{2 \lambda_{1}}|g(t)|_{2}^{2}+\frac{\lambda_{1}}{2}|u|_{2}^{2} .
$$

Using hypothesis $(H 1)$ and the fact that $\|u\|^{2} \geq(1 / 2)\|u\|^{2}+\left(\lambda_{1} / 2\right)|u|_{2}^{2}$, we have

$$
\frac{d}{d t}|u|_{2}^{2}+\lambda_{1}|u|_{2}^{2}+2 C_{1}|u|_{p}^{p}-2 C_{2}|\Omega| \leq \frac{1}{\lambda_{1}}|g(t)|_{2}^{2}
$$

Integrating (4.9) from $\tau$ to $r \in[\tau, t]$, we have

$$
|u(r)|_{2}^{2}+\lambda_{1} \int_{\tau}^{r}\|u(s)\|^{2} d s+2 C_{1} \int_{\tau}^{r}|u(s)|_{p}^{p} d s \leq|u(\tau)|_{2}^{2}+\frac{1}{\lambda_{1}} \int_{\tau}^{r}|g(s)|_{2}^{2} d s+2 C_{2}|\Omega|(t-\tau) .
$$

Thus,

$$
|u(r)|_{2}^{2}+\int_{\tau}^{r}\|u(s)\|^{2} d s+\int_{\tau}^{r}|u(s)|_{p}^{p} d s \leq C\left[|u(\tau)|_{2}^{2}+\int_{\tau}^{r}|g(s)|_{2}^{2} d s+(t-\tau)\right] .
$$

Multiplying (1.1) by $u_{t}$ then integrating over $\Omega$, we have

$$
\left|u^{\prime}(r)\right|_{2}^{2}+\frac{1}{2} \frac{d}{d r}\left(\|u(r)\|^{2}+2 \int_{\Omega} F(u(x, r)) d x\right) \leq \frac{1}{2}|g(r)|_{2}^{2}+\frac{1}{2}\left|u^{\prime}(r)\right|_{2}^{2} .
$$

Integrating now between $s \in[\tau, r]$ and $r \leq t$, we obtain

$$
\int_{s}^{r}\left|u^{\prime}(\theta)\right|_{2}^{2} d \theta+\|u(r)\|^{2}+\int_{\Omega} F(u(x, r)) d x \leq \int_{s}^{r}|g(\theta)|_{2}^{2} d \theta+\|u(s)\|^{2}+\int_{\Omega} F(u(x, s)) d x .
$$

From (4.13) and using hypothesis (H1), we get

$$
\begin{aligned}
& \int_{s}^{r}\left|u^{\prime}(\theta)\right|_{2}^{2} d \theta+\|u(r)\|^{2}+\alpha_{1} \int_{\Omega}|u(x, r)|^{p} d x-\alpha_{2}|\Omega| \\
& \leq\|u(s)\|^{2}+\int_{s}^{r}|g(\theta)|_{2}^{2} d \theta+\alpha_{3} \int_{\Omega}|u(x, r)|^{p} d x+\alpha_{4}|\Omega| .
\end{aligned}
$$


Hence

$$
\int_{s}^{r}\left|u^{\prime}(\theta)\right|_{2}^{2} d \theta+\|u(r)\|^{2}+\alpha_{1} u(r)_{p}^{p} \leq\|u(s)\|^{2}+\int_{s}^{t}|g(\theta)|_{2}^{2} d \theta+\alpha_{3}|u(s)|_{p}^{p}+\left(\alpha_{2}+\alpha_{4}\right)|\Omega| .
$$

Integrating inequality (4.15) with respect to $s$ from $\tau$ to $r$, we obtain

$$
\begin{aligned}
& (r-\tau)\left[\|u(r)\|^{2}+|u(r)|_{p}^{p}\right] \\
& \leq C\left[\int_{\tau}^{r}\|u(s)\|^{2} d s+\int_{\tau}^{r}|u(s)|_{p}^{p} d s\right]+C(t-\tau) \int_{\tau}^{t}|g(s)|_{2}^{2} d s+C|\Omega|(t-\tau),
\end{aligned}
$$

for all $t \geq \tau, r \in[\tau, t]$.

From (4.16) and (4.11), we obtain that

$$
\|u(r)\|^{2}+|u(r)|_{p}^{p} \leq C\left[\left|u_{\tau}\right|_{2}^{2}+\int_{\tau}^{\tau+2}|g(s)|_{2}^{2} d s+1\right]
$$

for all $r \in[\tau+1, \tau+2]$.

From (4.7), taking $t=\tau+3$ and $\epsilon=2$ we have

$$
\left|u^{\prime}(r)\right|_{2}^{2} \leq(4 l+3) \int_{\tau+1}^{\tau+3}\left|u^{\prime}(\theta)\right|_{2}^{2} d \theta+\int_{\tau+1}^{\tau+3}\left|g^{\prime}(\theta)\right|_{2}^{2} d \theta
$$

for all $r \in[\tau+2, \tau+3]$.

Analogously, and if we take $s=\tau+1$ and $r=t=\tau+3$ in inequlity (4.15), then

$$
\begin{aligned}
& \int_{\tau+1}^{\tau+3}\left|u^{\prime}(s)\right|_{2}^{2} d s+\|u(\tau+3)\|^{2}+\alpha_{1}|u(\tau+3)|_{p}^{p} \\
& \quad \leq\|u(\tau+1)\|^{2}+\int_{\tau}^{\tau+3}|g(s)|_{2}^{2} d s+\alpha_{3}|u(\tau+1)|_{p}^{p}+\left(\alpha_{2}+\alpha_{4}\right)|\Omega| .
\end{aligned}
$$

From (4.18) and (4.19), we obtain

$$
\begin{aligned}
\left|u^{\prime}(r)\right|_{2}^{2} \leq & (4 l+3)\left[\|u(\tau+1)\|^{2}+\alpha_{3}|u(\tau+1)|_{p}^{p}\right] \\
& +(4 l+3)\left[\left(\alpha_{2}+\alpha_{4}\right)|\Omega|+\int_{\tau}^{\tau+3}|g(s)|_{2}^{2} d s\right]+\int_{\tau+1}^{\tau+3}\left|g^{\prime}(\theta)\right|_{2}^{2} d \theta
\end{aligned}
$$

for all $r \in[\tau+2, \tau+3]$.

Owing to this inequality and (4.17), we have

$$
\left|u^{\prime}(r)\right|_{2}^{2} \leq C\left[\left|u_{\tau}\right|_{2}^{2}+\int_{\tau}^{\tau+3}\left[|g(s)|_{2}^{2}+\left|g^{\prime}(s)\right|_{2}^{2}\right] d s+1\right]
$$

for all $r \in[\tau+2, \tau+3]$. 
From (3.23), (3.60) and using Young's inequality, we have

$$
\|u(r)\|_{L^{2 p-2}(\Omega)}^{2 p-2}+\|u(r)\|_{S_{0}^{2}(\Omega)}^{2} \leq C\left(\left|u^{\prime}(r)\right|_{2}^{2}+\|u(r)\|^{2}+|u(r)|_{p}^{p}+1+|g(r)|_{2}^{2}\right)
$$

for all $r \geq \tau$.

From (4.22) and thank to (4.21), we have

$$
\begin{aligned}
\|u(r)\|_{L^{2 p-2}(\Omega)}^{2 p-2}+\|u(r)\|_{S_{0}^{2}(\Omega)}^{2} \leq & C\left[\left|u_{\tau}\right|_{2}^{2}+\int_{\tau}^{\tau+3}\left[|g(s)|_{2}^{2}+\left|g^{\prime}(s)\right|_{2}^{2}\right] d s+1\right] \\
& +C\left(\|u(r)\|^{2}+|u(r)|_{p}^{p}+|g(r)|_{2}^{2}\right)
\end{aligned}
$$

for all $r \in[\tau+2, \tau+3]$. From (4.23) and thanks to (4.17), we have

$$
\|u(r)\|_{L^{2 p-2}(\Omega)}^{2 p-2}+\|u(r)\|_{S_{0}^{2}(\Omega)}^{2} \leq C\left[\left|u_{\tau}\right|+\int_{\tau}^{\tau+3}\left[|g(s)|_{2}^{2}+\left|g^{\prime}(s)\right|_{2}^{2}\right] d s+1+\sup _{r \in[\tau+2, \tau+3]}|g(r)|_{2}^{2}\right],
$$

for all $r \in[\tau+2, \tau+3]$. Now, observe that by Cauchy's inequality

$$
|g(r)| \geq|g(\tau+2)|+\left(\int_{\tau+2}^{\tau+3}\left|g^{\prime}(s)\right|_{2}^{2} d s\right)^{1 / 2}
$$

for all $r \in[\tau+2, \tau+3], \tau \in \mathbb{R}, u_{\tau} \in L^{2}(\Omega)$.

Thus, from (4.23), we have

$$
\begin{aligned}
& \left\|U(\tau+2, \tau) u_{\tau}\right\|_{L^{2 p-2}(\Omega)}^{2 p-2}+\left\|U(\tau+2, \tau) u_{\tau}\right\|_{S_{0}^{2}(\Omega)}^{2} \\
& \quad \leq C\left[\left|u_{\tau}\right|_{2}^{2}+\int_{\tau}^{\tau+3}\left(|g(s)|_{2}^{2}+\left|g^{\prime}(s)\right|_{2}^{2}\right) d s+1+|g(\tau+2)|_{2}^{2}\right] .
\end{aligned}
$$

for all $\tau \in \mathbb{R}, u_{\tau} \in L^{2}(\Omega)$. From this inequlity, and the fact that $\mathcal{A}(\tau)=U(\tau, \tau-2) A(\tau-2)$, we obtain

$$
\|v\|_{L^{2 p-2}(\Omega)}^{2 p-2}+\|v\|_{S_{0}^{2}(\Omega)}^{2} \leq C\left[\sup _{w \in A(\tau-2)}|w|_{2}^{2}+\int_{\tau-2}^{\tau+1}\left(|g(s)|_{2}^{2}+\left|g^{\prime}(s)\right|_{2}^{2}\right) d s+1+|g(\tau)|_{2}^{2}\right] .
$$

for all $v \in A(\tau)$, and any $\tau \in \mathbb{R}$. Now, thank to (4.2), (4.3), we obtain (4.3) from (4.27).

\section{Acknowledgment}

The authors thanks the reviewers very much for valuable comments and suggestions. 


\section{References}

[1] V. V. Grušin, "A certain class of elliptic pseudodifferential operators that are degenerate on a submanifold," Matematicheskii Sbornik, vol. 84, pp. 163-195, 1971, English translation in: Mathematics of the USSR-Sbornik, vol. 13, pp. 155-183, 1971.

[2] C. T. Anh, "Pullback attractors for non-autonomous parabolic equations involving Grushin operators," Electronic Journal of Differential Equations, vol. 2010, pp. 1-14, 2010.

[3] C. T. Anh, P. Q. Hung, T. D. Ke, and T. T. Phong, "Global attractor for a semilinear parabolic equation involving Grushin operator," Electronic Journal of Differential Equations, no. 32, pp. 1-11, 2008.

[4] C. T. Anh and T. D. Ke, "Existence and continuity of global attractors for a degenerate semilinear parabolic equation," Electronic Journal of Differential Equations, vol. 2009, no. 61, pp. 1-13, 2009.

[5] C. T. Anh and N. V. Quang, "Uniform attractors for a non-autonomous parabolic equation involving Grushin operator," Acta Mathematica Vietnamica, vol. 36, no. 1, pp. 19-33, 2011.

[6] C. T. Anh and T. V. Manh, "On the dynamics of nonautonomous parabolic systems involving the Grushin operators," International Journal of Mathematics and Mathematical Sciences, vol. 2011, Article ID 178057, 27 pages, 2011.

[7] N. D. Binh, "Global attractors for degenerate parabolic equations without uniqueness," International Journal of Mathematical Analysis, vol. 4, no. 21-24, pp. 1067-1078, 2010.

[8] Q. Ma, S. Wang, and C. Zhong, "Necessary and sufficient conditions for the existence of global attractors for semigroups and applications," Indiana University Mathematics Journal, vol. 51, no. 6, pp. 1541 1559, 2002.

[9] C.-K. Zhong, M.-H. Yang, and C.-Y. Sun, "The existence of global attractors for the norm-to-weak continuous semigroup and application to the nonlinear reaction-diffusion equations," Journal of Differential Equations, vol. 223, no. 2, pp. 367-399, 2006.

[10] C. T. Anh, T. Q. Bao, and L. T. Thuy, "Regularity and fractal dimension estimates of pullback attractors for a non-autonomous semilinear degenerate parabolic equation," , submitted.

[11] M. Anguiano, T. Caraballo, and J. Real, "An exponential growth condition in $H^{2}$ for the pullback attractor of a non-autonomous reaction-diffusion equation," Nonlinear Analysis, vol. 72, no. 11, pp. 4071-4075, 2010.

[12] M. Anguiano, T. Caraballo, and J. Real, " $H^{2}$-boundedness of the pullback attractor for a nonautonomous reaction-diffusion equation," Nonlinear Analysis, vol. 72, no. 2, pp. 876-880, 2010.

[13] Y. Li and C. Zhong, "Pullback attractors for the norm-to-weak continuous process and application to the nonautonomous reaction-diffusion equations," Applied Mathematics and Computation, vol. 190, no. 2, pp. 1020-1029, 2007.

[14] G. Łukaszewicz, "On pullback attractors in $L^{p}$ for nonautonomous reaction-diffusion equations," Nonlinear Analysis, vol. 73, no. 2, pp. 350-357, 2010.

[15] Y. Wang and C. Zhong, "On the existence of pullback attractors for non-autonomous reactiondiffusion equations," Dynamical Systems, vol. 23, no. 1, pp. 1-16, 2008.

[16] N. T. C. Thuy and N. M. Tri, "Some existence and nonexistence results for boundary value problems for semilinear elliptic degenerate operators," Russian Journal of Mathematical Physics, vol. 9, no. 3, pp. 365-370, 2002.

[17] J. C. Robinson, Infinite-Dimensional Dynamical Systems, Cambridge Texts in Applied Mathematics, Cambridge University Press, Cambridge, UK, 2001. 


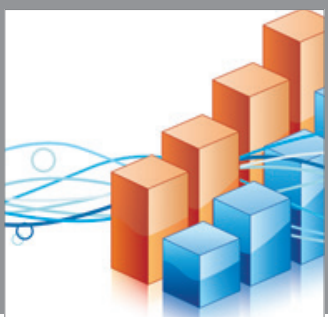

Advances in

Operations Research

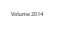

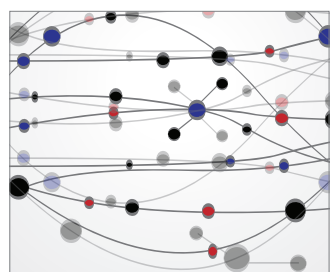

\section{The Scientific} World Journal
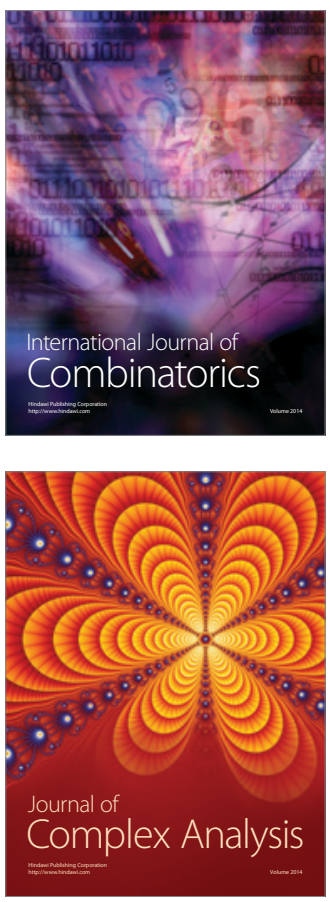

International Journal of

Mathematics and

Mathematical

Sciences
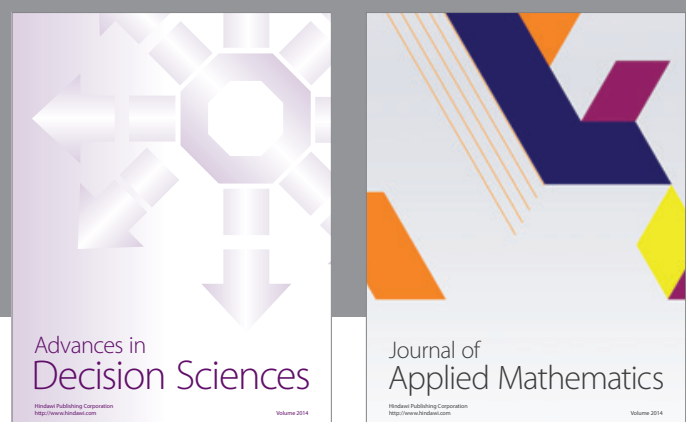

Journal of

Applied Mathematics
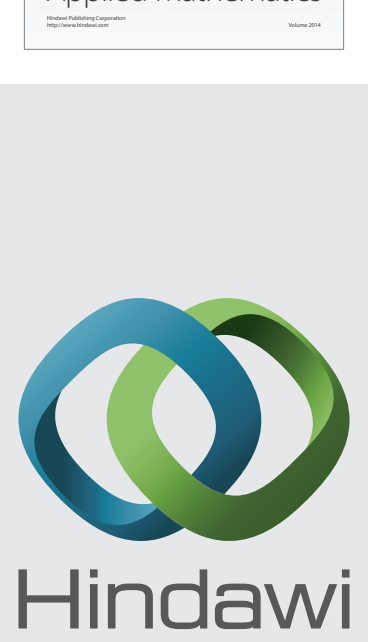

Submit your manuscripts at http://www.hindawi.com
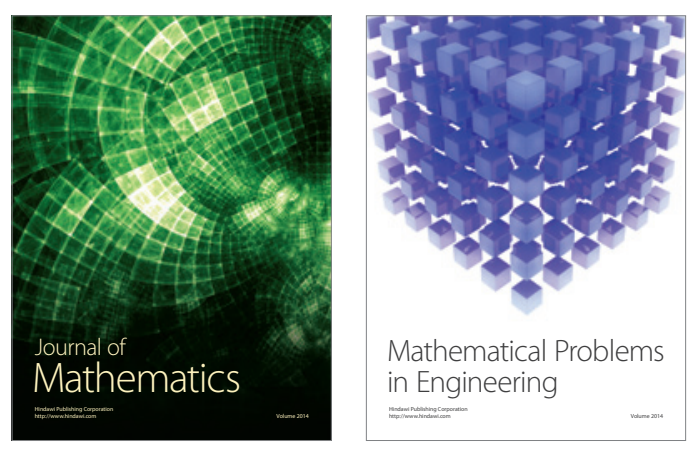

Mathematical Problems in Engineering
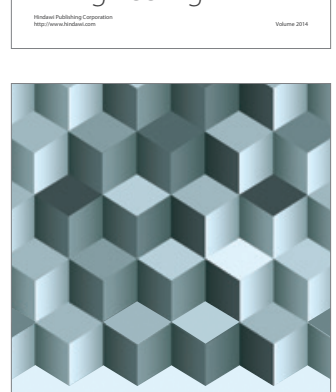

Journal of

Function Spaces
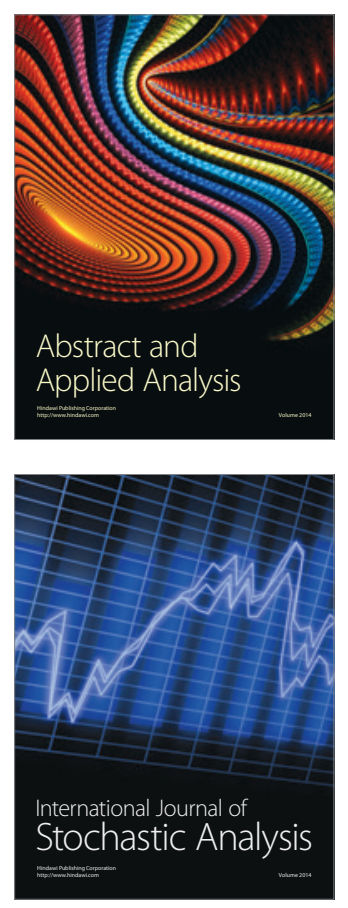

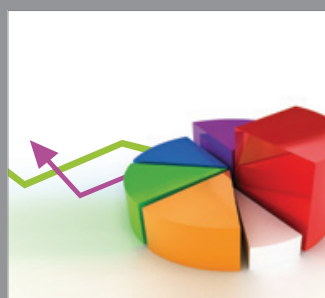

ournal of

Probability and Statistics

Promensencen
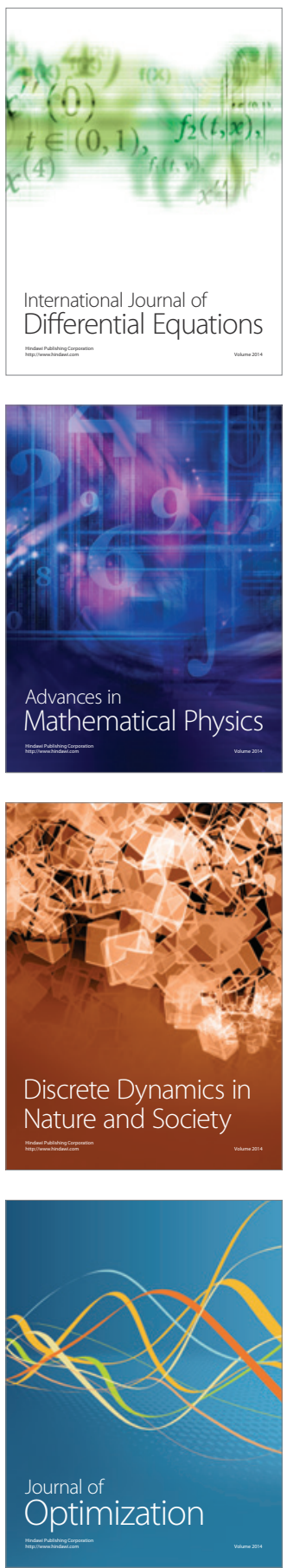